Arq. Bras. Med. Vet. Zootec., v.60, n.1, p.58-65, 2008

\title{
Efeitos da progesterona exógena sobre o desenvolvimento folicular em ovelhas
}

\author{
[Effects of exogen progesterone on follicular development in sheep] \\ L.F. Uribe-Velásquez ${ }^{1}$, E. $O b a^{2}$, M.I.L. Souza ${ }^{3}$ \\ ${ }^{1}$ Departamento de Salud Animal - Universidad de Caldas \\ Calle $65 n^{\circ} 26-10$ \\ Manizales - Colombia \\ ${ }^{2}$ Faculdade de Medicina Veterinária e Zootecnia - UNESP - Botucatu, SP \\ ${ }^{3}$ Departamento de Morfofisiologia - UFMS - Campo Grande, MS
}

\begin{abstract}
RESUMO
Avaliaram-se os efeitos da progesterona $\left(\mathrm{P}_{4}\right)$ sobre o crescimento folicular e na endocrinologia reprodutiva em ovelhas Bergamácia. Quatorze ovelhas sincronizadas com prostaglandinas $\left(\mathrm{PGF}_{2 \alpha}\right)$ foram distribuídas em dois grupos (n=7/grupo): grupo-controle e grupo tratado com progesterona (CIDR) depois da ovulação (dia zero). Desde o dia anterior à aplicação de PG até o dia 10, realizaram-se monitoramentos ultra-sonográficos para estabelecer o crescimento folicular. Amostras de sangue foram colhidas para a determinação de $\mathrm{P}_{4}$ desde o dia anterior à aplicação de $\mathrm{PG}$ até o dia 10 depois da ovulação. Para o perfil dos pulsos de hormônio luteinizante (LH), as colheitas de sangue ocorreram em intervalos de 30 minutos por um período de oito horas, nos dias um e seis. As taxas de crescimento diferiram $(\mathrm{P}<0,001)$ entre os grupos, $0,91 \pm 0,15$ e $0,70 \pm 0,16 \mathrm{~mm} /$ dia para os grupos controle e tratado, respectivamente. Os dias do platô dos animais controle e tratados foram de $1,9 \pm 0,72$

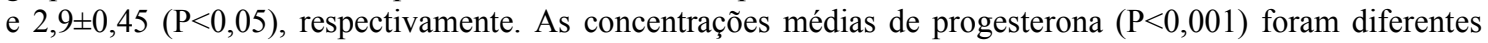
entre os tratamentos. A freqüência dos pulsos diferiu no primeiro dia do ciclo $(\mathrm{P}<0,01)$, com valores de $2,55 \pm 0,09 \mathrm{pulsos} / 8$ horas no grupo-controle e de $1,49 \pm 0,11 \mathrm{pulsos} / 8$ horas no grupo tratado. No sexto dia, o grupo-controle $2,20 \pm 0,09$ pulsos/8 horas apresentou maior número de pulsos $(\mathrm{P}<0,05)$ que o grupo tratado, $1,22 \pm 0,11$ pulsos/ 8 horas. Os efeitos inibitórios da progesterona exógena no diâmetro do folículo dominante foram mediados pela redução na freqüência dos pulsos de LH.
\end{abstract}

Palavras-chave: ovelha, dinâmica folicular, LH, ovulação, progesterona

\begin{abstract}
The effects of progesterone $\left(P_{4}\right)$ on ovarian follicular growth and reproductive endocrinology were studied. Fourteen ewes, synchronized using prostaglandin $\left(P G F_{2 \alpha}\right)$, were randomly divided in two groups ( $n=7 /$ group $)$; control group and progesterone-treated group (CIDR) after ovulation (day zero). From one day before PG injection until day 10, daily ultrasonic examinations were done to establish follicular growth. Blood samples for $\mathrm{P}_{4}$ plasma concentration determinations were collected from one day before PG until day 10 post-ovulation. For profile of LH pulses, blood samples were collected at 30-min intervals for a period of $8 \mathrm{~h}$ on days one and six. The growth rate was different between groups $(P<0.001)$, being $0.91 \pm 0.15$ and $0.70 \pm 0.16 \mathrm{~mm} / \mathrm{d}$ for control and treated-group, respectively. The lengthened of the static phase for control and treated group were $1.9 \pm 0.72$ and $2.9 \pm 0.45 d(P<0.05)$, respectively. Mean concentrations of $P_{4}(P<0.001)$ were different between treatments. Differences in LH pulse frequency on day one $(P<0.01)$ were observed with values of $2.55 \pm 0.09$ pulses $/ 8 \mathrm{~h}$ in control group and $1.49 \pm 0.11$ pulses/8h in treated-group. On day six, control group showed higher values for LH pulse frequency (2.20 0.09 pulses $/ 8 h)$ than treated-group $(P<0.05)$, (1.22 \pm 0.11 pulses $/ 8 h)$. In conclusion, the inhibitory effects of exogen $\mathrm{P}_{4}$ on the diameter of dominant follicle was mediated by reduced LH pulse frequency.
\end{abstract}

Keywords: sheep, follicular dynamic, LH, ovulation, progesterone

Recebido em 1 de agosto de 2006

Aceito em 17 de dezembro de 2007

E-mail: lfuribe@ucaldas.edu.co 


\section{INTRODUÇÃO}

Em ovelhas, a emergência folicular ocorre tanto no início da fase folicular quanto durante a fase luteínica do ciclo, mecanismo controlado pelo FSH hipofisário. Apenas os folículos maiores que $2 \mathrm{~mm}$ podem ser recrutados, enquanto os folículos menores são menos sensíveis ao recrutamento (Driancourt et al., 1985). A hipófise anterior secreta o hormônio luteinizante (LH) em resposta ao hormônio liberador de gonadotrofinas (GnRH), o qual é liberado no fluxo portal-hipofisário pelo hipotálamo (Goodman e Karsch, 1980). Os mecanismos do cérebro que modulam a liberação pulsátil da secreção de GnRH que, por sua vez, induzem os pulsos secretórios de LH apresentam papel importante na manutenção da ciclicidade. A freqüência dos pulsos de GnRH/LH é bem mais elevada na fase folicular que durante a fase lútea, aumento que contribui para o desenvolvimento folicular ovariano (Tsukamura, 1998).

As mudanças na freqüência e na amplitude dos pulsos são, primariamente, um reflexo da intensa ação dos esteróides sexuais (Goodman e Karsch, 1980). Em ovelhas ovariectomizadas que receberam implantes subcutâneos de estradiol $\left(E_{2}\right)$, a freqüência dos pulsos de $\mathrm{LH}$ variou inversamente à quantidade de hormônio presente no implante. $\mathrm{O}$ índice de secreção dos pulsos de LH depende, também, da época, pois os efeitos inibitórios de uma dose de $\mathrm{E}_{2}$ são mais baixos durante a estação reprodutiva que na época do anestro. Essa resposta de $\mathrm{E}_{2}$ durante a estação reprodutiva é restaurada em ovelhas ovariectomizadas quando tratadas com progesterona $\left(\mathrm{P}_{4}\right)$, explicando, assim, a ação sinérgica desses dois hormônios esteroidais. Essa variação sazonal na curva de dose-resposta de $E_{2}$ e de LH, ligado ao sinergismo de $\mathrm{P}_{4}$, pode explicar a maioria das mudanças da secreção pulsátil de LH observadas em ovelhas intactas (Thiéry e Martin, 1991).

Evidências mostram que, na ovelha, a principal atuação da P4 ocorre no hipotálamo, decrescendo a freqüência dos pulsos de $\mathrm{LH}$ e aumentando sua amplitude, enquanto $\mathrm{o} \mathrm{E}_{2}$ atua na hipófise, diminuindo a amplitude dos pulsos sem afetar a freqüência (Goodman e Karsch, 1980; Goodman et al., 1982).
A freqüência dos pulsos de LH é a variável que mais reflete, consistentemente, o comportamento do hormônio, enquanto as outras variáveis referentes à secreção de $\mathrm{LH}$, concentração média e amplitude dos pulsos podem ser confundidas com as mudanças resultantes na amplitude dos pulsos de GnRH e na resposta hipofisária. O objetivo principal deste estudo foi determinar, por meio de acompanhamento ultra-sonográfico, o efeito da $\mathrm{P}_{4}$ exógena no crescimento do folículo dominante e, por meio de radioimunoensaio (RIA), a concentração plasmática de $\mathrm{LH}$ e da $\mathrm{P}_{4}$ em ovelhas Bergamácia.

\section{MATERIAL E MÉTODOS}

Utilizaram-se 14 animais da raça Bergamácia, durante a estação reprodutiva, com peso corporal médio de $60,42 \pm 8,16$ e de $61,56 \pm 6,54 \mathrm{~kg}$ para os tratamentos 1 (controle) e 2 (CIDR), respectivamente, e com idades médias de 4,5 $\pm 1,2$ e de $4 \pm 0,9$ anos na mesma ordem de citação. Os animais, previamente examinadas quanto ao estado clínico geral, sanitário e reprodutivo, foram mantidos em boxes de $3 \times 3 \mathrm{~m}$, sob luminosidade natural.

Submetidas a um período pré-experimental de um mês, para adaptação à alimentação e à estabulação, as ovelhas foram inicialmente pesadas e alimentadas com ração concentrada ( $20 \%$ de proteína bruta e $5 \%$ de fibra bruta) e feno de Cynodon dactylon (L.) Pers. cv "coast cross" (5\% de proteína bruta e $35,9 \%$ de fibra bruta, na matéria seca); a quantidade formada foi de $1,5 \mathrm{~kg} /$ cabeça/dia de ração concentrada e $2,0 \mathrm{~kg} / \mathrm{cabeça} / \mathrm{dia}$ de feno e mistura mineral e água à vontade.

Em todas as ovelhas, administraram-se, via intramuscular, duas doses de um análogo sintético de prostaglandina- $\mathrm{PGF}_{2 \alpha}, \quad 125 \mu \mathrm{g}$ $\left(\operatorname{Ciosin}^{\mathrm{R}}\right)^{1}$, com intervalo de sete dias, durante a metade da fase lútea, para a sincronização de estro. Posteriormente, foram distribuídas, aleatoriamente, em dois grupos: grupo-controle $(n=7)$ e grupo tratado $(n=7)$ com progesterona ${ }^{2}$ contendo $0,3 \mathrm{~g}$ de progesterona e inserido intravaginalmente após a ovulação (6-10 horas).

\footnotetext{
${ }^{1}$ Mallinckrodt Veterinary - São Paulo, Brasil.

${ }^{2}$ CIDR, Controlled internal drug release, AHÍ Plastic

Moulding Company, Hamilton - Nova Zelândia.
} 
Desde o dia anterior à aplicação da segunda dose de $\mathrm{PGF}_{2 \alpha}$ até o $10^{\circ}$ dia do ciclo, as ovelhas foram monitoradas por meio de ultra-som. O dia da ovulação foi definido como o momento do desaparecimento do maior folículo ( $\geq 5 \mathrm{~mm})$. As ovelhas em posição de estação foram monitoradas por meio de ultra-som provido de um transductor linear prostático (SSD-500) ${ }^{3}$. Todos os folículos $\geq 2 \mathrm{~mm}$ foram monitorados e mapeados diariamente. Para a análise do dia de emergência folicular, foram incluídos apenas os folículos que atingiram pelo menos, $4 \mathrm{~mm}$ de diâmetro, e identificados no mínimo durante quatro dias, quando apresentaram crescimento de, em média, 1mm/dia (Ravindra et al., 1994). A duração do crescimento foi definida como o número de dias entre a primeira mensuração do folículo dominante e o dia de cessação de seu crescimento. A taxa de crescimento folicular foi calculada pela diferença entre o maior e o menor diâmetro detectável pela ultra-sonografia, dividido pelo número de dias em crescimento.
Denominou-se de fase estática o número de dias de permanência com o diâmetro máximo. O dia do início da regressão (atresia) foi identificado como o último dia da fase estática (platô), a partir do qual ocorreu a diminuição progressiva do diâmetro do folículo. $\mathrm{O}$ corpo lúteo foi observado desde o terceiro dia após a ovulação, e foi monitorado e mapeado diariamente.

Foram colhidas amostras de sangue, sempre às $8 \mathrm{~h}$, por venopunção jugular, antes da aplicação de PG e durante todo o período experimental. Para a quantificação do LH, colheram-se 17 amostras de sangue no primeiro e 17 no sexto dia do ciclo estral, durante oito horas, seguidas em intervalos de 30 minutos, para definir sua amplitude e freqüência, em tubos heparinizados, que foram centrifugados a $1000 \mathrm{xg}$ durante 15 minutos; o plasma armazenado a $-20^{\circ} \mathrm{C}$ foi usado para determinação da $\mathrm{P}_{4}$ e do $\mathrm{LH}$. No diagrama que se segue, apresentam-se os esquemas de tratamentos dos grupos.

Grupo 1 (controle):

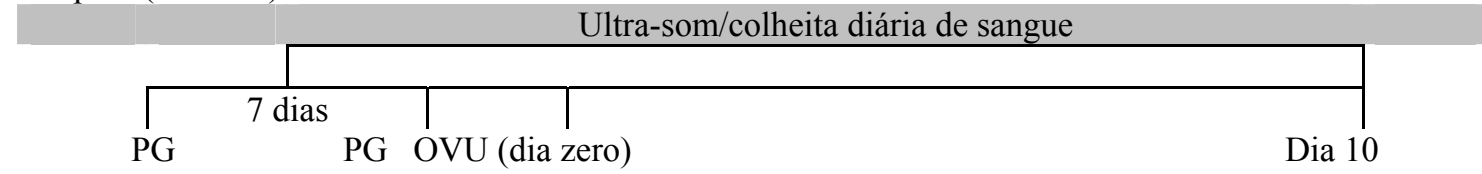

Grupo 2 (tratado com progesterona exógena - CIDR):

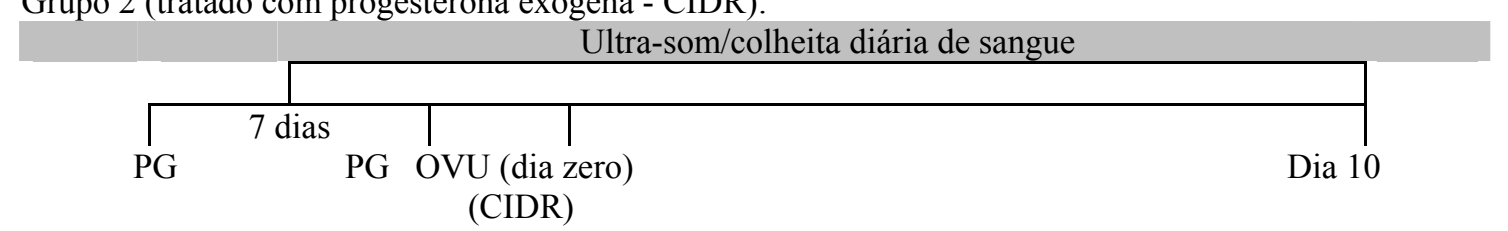

As concentrações plasmáticas de $\mathrm{P}_{4}$ foram determinadas por RIA, utilizando-se kits comerciais Coat-A-Count ${ }^{4}$ em fase sólida. A sensibilidade do ensaio para $\mathrm{P}_{4}$ foi de $0,1 \mathrm{ng} / \mathrm{ml}$. Para determinação do LH, utilizou-se o procedimento recomendado por Forsberg et al. (1993), usando um anticorpo monoclonal (Mab 518B7), com sensibilidade mínima de $0,3 \mu \mathrm{g} / \mathrm{l}$. O pulso foi definido como a maior concentração observada quando comparada com a média mais um desvio-padrão.

Para a concentração plasmática de $\mathrm{P}_{4}$, foram utilizados três controles intra-ensaio com baixa

\footnotetext{
${ }^{3}$ Modelo SSD-500 e UST-660-7,5, Aloka Co., Ltda. - Tokio, Japão.

${ }^{4}$ Diagnostic Products Corporation - Los Angeles, EUA.
}

$(0,41 \pm 0,02 \mathrm{ng} / \mathrm{ml})$, média $(2,27 \pm 0,10 \mathrm{ng} / \mathrm{ml})$ e alta $(5,97 \pm 0,13 \mathrm{ng} / \mathrm{ml})$ concentração de $\mathrm{P}_{4}$. Os coeficientes de variação inter-ensaio foram: $10,5 \%(0,38 \pm 0,04 \mathrm{ng} / \mathrm{ml}) ; 2,2 \%(2,31 \pm 0,05 \mathrm{ng} / \mathrm{ml})$ e $2,3 \%(6,07 \pm 0,14 \mathrm{ng} / \mathrm{ml})$ para $\mathrm{P}_{4}$, na mesma ordem de citação anterior. Para a dosagem de $\mathrm{LH}$, foram realizados dois ensaios, que apresentaram os coeficientes de variação intraensaio de $7,4 \% \quad(2,39 \pm 0,29 \mu \mathrm{g} / 1), \quad 2,7 \%$ $(4,37 \pm 0,03 \mu \mathrm{g} / \mathrm{l})$ e $19,6 \% \quad(7,6 \pm 0,14 \mu \mathrm{g} / \mathrm{l})$. Os coeficientes de variação inter-ensaio foram de $7,1 \%(2,42 \pm 0,04 \mu \mathrm{g} / 1), 13,4 \%(4,26 \pm 0,16 \mu \mathrm{g} / 1)$ e $17 \%(6,4 \pm 1,7 \mu \mathrm{g} / \mathrm{l})$, sempre na ordem citada.

Os efeitos dos tratamentos sobre as concentrações de $\mathrm{P}_{4}$ e de $\mathrm{LH}$ foram determinados pela análise de medidas repetidas, utilizando-se o 
Statistical Analysis System (Latour e Littell, 1996). As concentrações basais de LH e a amplitude dos pulsos foram transformadas em raiz quadrada. Para a análise da freqüência dos pulsos, os valores foram transformados em Log. Para os efeitos fixos (tratamento, dia, tratamento $\mathrm{x}$ dia), foi utilizado $\mathrm{o}$ teste $\mathrm{F}$, e, para as comparações múltiplas das médias, usou-se o teste Tukey-kramer. O teste não paramétrico de Wilcoxon-Mann-Whitney foi utilizado para as seguintes variáveis do folículo dominante da primeira onda folicular: 1) dia de emergência; 2) dia do diâmetro máximo; 3) diâmetro máximo e 4) dias na fase estática. Para a análise da taxa de crescimento, utilizou-se a análise de variância.

\section{RESULTADOS E DISCUSSÃO}

$\mathrm{Na}$ primeira onda de crescimento folicular, observou-se o desenvolvimento simultâneo de um grupo de folículos antrais, os quais provinham de um pool de folículos previamente recrutados no início da fase antral, confirmando as observações feitas por Fortune (1994). A primeira onda folicular caracterizou-se pelo desenvolvimento de um folículo grande, dominante, e uma quantidade variável de folículos pequenos, não dominantes. Essas observações assemelham-se às de Kruip e Brand (1975), que utilizaram peças de matadouro, de Rubianes et al. (1996), que empregaram laparoscopia, e de Leyva et al. (1998) que utilizaram a ultra-sonografia.

O perfil de crescimento do folículo dominante durante essa primeira onda foi diferente nas diferentes variáveis nos animais tratados com $\mathrm{P}_{4}$ exógena quando comparados com os do tratamento-controle (Tab. 1).

O dia de emergência da primeira onda folicular não diferiu $(\mathrm{P}>0,05)$ entre os dois grupos experimentais, respectivamente, valores próximos aos obtidos por Leyva et al. (1998) e menores que os de Ravindra et al. (1994).

Tabela 1. Folículo dominante da primeira onda folicular em ovelhas Bergamácia tratadas ou não com progesterona exógena (CIDR) no dia da ovulação (médias \pm EP)

\begin{tabular}{lcc}
\hline Variável & Controle & Tratadas- $\mathrm{P}_{4}$ \\
\hline Dia de emergência & $-0,71 \pm 0,73 \mathrm{a}$ & $0,0 \pm 0,64 \mathrm{a}$ \\
Dia do máximo diâmetro & $5,4 \pm 0,92 \mathrm{a}$ & $5,7 \pm 0,86 \mathrm{a}$ \\
Diâmetro máximo (mm) & $5,5 \pm 0,52 \mathrm{a}$ & $4,0 \pm 0,47 \mathrm{~b}$ \\
Dias do platô & $1,9 \pm 0,72 \mathrm{c}$ & $2,9 \pm 0,45 \mathrm{~d}$ \\
Taxa de crescimento (mm/dia) & $0,91 \pm 0,15 \mathrm{a}$ & $0,7 \pm 0,16 \mathrm{~b}$ \\
\hline
\end{tabular}

Letras distintas na linha representam diferenças significativas: a vs b $(\mathrm{P}<0,001)$; c vs d $(\mathrm{P}<0,05)$

$\mathrm{EP}=$ erro-padrão da média.

$\mathrm{O}$ folículo dominante atingiu seu máximo diâmetro, 5,5mm, no dia 5,4 nos animais do grupo-controle, e 4,0mm no dia 5,7 nos submetidos à $\mathrm{P}_{4}$ exógena. $\mathrm{A}$ diferença entre tratamentos só foi significativa para o diâmetro folicular $(\mathrm{P}<0,001)$. $\mathrm{O}$ diâmetro máximo observado no tratamento-controle foi semelhante ao verificado por Rubianes et al. (1996) e menor que o observado por Leyva et al. (1998), sugerindo possíveis diferenças entre raças.

De modo geral, a $\mathrm{P}_{4}$ tem sido associada com a supressão do crescimento folicular e ovulação, via eixo hipotalâmico-hipofisário-ovariano, possivelmente por atuar diretamente no ovário (Noel et al., 1994). In vivo, a $\mathrm{P}_{4}$ exógena retarda o desenvolvimento folicular em ratas e, in vitro, apresenta efeito inibitório irreversível na secreção de $E_{2}$ pelas células da granulosa (Karsch et al., 1977).

A administração do CIDR no dia da ovulação inibiu o folículo dominante, indicado pela diminuição no diâmetro folicular. Além disso, e segundo Ginther (1971), a $\mathrm{P}_{4}$ pode ter exercido efeito inibitório no folículo dominante. Ainda de acordo com o autor, esse mecanismo pode ser observado quando o hormônio é administrado tanto no início quanto no final do ciclo estral.

Os dias do platô diferiram entre os tratamentos $(\mathrm{P}<0,05)$, de forma semelhante ao que foi observado por Rubianes et al. (1996). Assim, o folículo dominante aumentou sua fase de crescimento para atingir seu diâmetro máximo, o que pode ocasionar mudanças no fluido folicular, 
comprometendo o desenvolvimento dos oócitos no final do ciclo.

A taxa de crescimento foi diferente $(\mathrm{P}<0,001)$ entre os dois grupos de animais. Estes resultados assemelham-se aos obtidos por Ravindra et al. (1994), Rubianes et al. (1996) e Leyva et al. (1998). Taxas de crescimento folicular de 0,9 a $1,7 \mathrm{~mm} /$ dia foram verificadas em diferentes raças ovinas por Castonguay et al. (1990). As taxas verificadas no presente estudo foram menores que aquelas encontradas por Driancourt e Cahill (1984), os quais constataram taxa de crescimento de $1,6 \mathrm{~mm} /$ dia nos folículos durante a fase folicular, monitorados por laparotomia. Desse modo, pode-se afirmar que o crescimento do folículo dominante da primeira onda de desenvolvimento folicular nas fêmeas ovinas foi inibido pela alta concentração plasmática de $\mathrm{P}_{4}$, obtida pela inserção do CIDR após a ovulação. A $\mathrm{P}_{4}$ exerce um feedback negativo no hipotálamo e hipófise, regulando a liberação das gonadotrofinas e, principalmente, na liberação de
LH. Assim, os efeitos inibitórios de $\mathrm{P}_{4}$ no crescimento do folículo dominante não ovulatório podem ter sido mediados pelas mudanças na liberação de LH (Martin, 1984). A $\mathrm{P}_{4}$ facilita também a apertura dos canais de cloro na célula, prolongando, então, a ação inibitória do GABA nos neurônios (Klein e Herzog, 1998). No presente estudo, possivelmente a concentração plasmática de $\mathrm{P}_{4}$ foi suficientemente elevada para suprimir a freqüência dos pulsos de LH.

A média \pm erro-padrão da concentração basal $(\mu \mathrm{g} / \mathrm{l})$, a amplitude dos pulsos $(\mu \mathrm{g} / \mathrm{l})$ e a freqüência dos pulsos (pulsos/8 horas) de LH são apresentadas na Tab. 2. A concentração plasmática basal de LH no primeiro dia do ciclo estral (dia zero = ovulação) não foi diferente $(\mathrm{P}>0,05)$ entre os grupos. Também não se verificaram diferenças entre tratamentos no sexto dia do ciclo estral.

Tabela 2. Médias \pm erro-padrão da média da concentração basal plasmática ( $\mu \mathrm{g} / \mathrm{l})$, amplitude ( $\mu \mathrm{g} / \mathrm{l})$ e freqüência dos pulsos (pulsos/8h) do hormônio luteinizante no primeiro e sexto dias do ciclo estral em fêmeas ovinas Bergamácia tratadas ou não com $\mathrm{P}_{4}$ exógena (CIDR) no dia da ovulação (dia zero)

\begin{tabular}{ccccc}
\hline $\begin{array}{c}\text { Dia do } \\
\text { ciclo estral }\end{array}$ & Grupo & $\begin{array}{c}\text { Concentração basal } \\
(\mu \mathrm{g} / \mathrm{l})\end{array}$ & $\begin{array}{c}\text { Amplitude dos pulsos } \\
(\mu \mathrm{g} / \mathrm{l})\end{array}$ & $\begin{array}{c}\text { Freqüência dos pulsos } \\
(\mathrm{pulsos} / 8 \mathrm{~h})\end{array}$ \\
\hline \multirow{2}{*}{1} & Controle & $0,66 \pm 0,11 \mathrm{a}$ & $0,33 \pm 0,30 \mathrm{a}$ & $2,55 \pm 0,09 \mathrm{a}$ \\
& Tratado & $0,56 \pm 0,27 \mathrm{a}$ & $0,42 \pm 0,21 \mathrm{a}$ & $1,49 \pm 0,11 \mathrm{~b}$ \\
6 & Controle & $0,68 \pm 0,11 \mathrm{~A}$ & $0,87 \pm 0,30 \mathrm{~A}$ & $2,20 \pm 0,09 \mathrm{~A}$ \\
& Tratado & $0,58 \pm 0,27 \mathrm{~A}$ & $0,70 \pm 0,21 \mathrm{~A}$ & $1,22 \pm 0,11 \mathrm{~B}$ \\
\hline
\end{tabular}

Médias com distintas letras na coluna diferiram entre si: a vs b $(\mathrm{P}<0,01)$ e $\mathrm{A}$ vs $\mathrm{B}(\mathrm{P}<0,05)$.

Semelhante aos resultados mostrados por Campbell et al. (1990), as concentrações basais de LH não apresentaram variações significativas durante o ciclo estral. Não ocorreram mudanças nos dias que se seguiram ao tratamento nos animais tratados com o CIDR no dia da ovulação. Tem sido apontado que os efeitos produzidos pela $\mathrm{P}_{4}$ exógena na concentração basal de LH podem ser controlados por outros mecanismos, tais como: atividade das células lúteas; fluxo sangüíneo local e ocitocina originada das células grandes lúteas (Alecozay et al., 1988). Entretanto, a freqüência dos pulsos de LH é a variável que mais reflete, consistentemente, o comportamento do hormônio (Thiéry e Martin, 1991).
A amplitude dos pulsos de LH não diferiu $(\mathrm{P}>0,05)$ entre os grupos no primeiro e no sexto dias do ciclo estral. Entretanto, a $\mathrm{P}_{4}$ exógena diminuiu $(\mathrm{P}<0,05)$ a amplitude dos pulsos no primeiro dia quando comparada àquela provocada no sexto dia. A amplitude dos pulsos foi menor que os valores apresentados por Haresign et al. (1995) e similar às verificadas por Currie et al. (1993). Norris et al. (1989) não constataram mudanças na amplitude dos pulsos entre as diferentes fases do ciclo estral. As mudanças observadas na amplitude dos pulsos são, primariamente, um reflexo da intensa atividade dos esteróides sexuais (Goodman e Karsch, 1980). Goodman et al. (1982) observaram que a ação principal da $\mathrm{P}_{4}$ é no hipotálamo, ao diminuir a freqüência dos pulsos e aumentar sua amplitude. 
A freqüência dos pulsos diferiu $(\mathrm{P}<0,01)$ entre os dois grupos de animais no primeiro e sexto dias do ciclo estral. Verificou-se, então, que o dispositivo contendo $\mathrm{P}_{4}$ gerou aumento na concentração plasmática de $\mathrm{P}_{4}$, característicos da fase lútea, alterando a freqüência pulsátil de $\mathrm{LH}$ e a atividade folicular. Assim, essa concentração plasmática de $\mathrm{P}_{4}$ permitiu que $\mathrm{o}$ folículo dominante não fosse atingido pela atresia normal e mantivesse seu crescimento e dominância por tempo mais prolongado.

A freqüência pulsátil obtida no primeiro dia foi menor que a relatada por McNatty et al. (1984). A freqüência dos pulsos encontrada no sexto dia foi semelhante à observada por Downing et al. (1997), e as do primeiro e sexto dias constatadas no grupo tratado com a $\mathrm{P}_{4}$ exógena foram semelhantes às obtidas por Leyva et al. (1998), ao trabalharem com ovelhas Suffolk.

Constataram-se mudanças na freqüência dos pulsos nos diferentes dias após a ovulação. Assim, a freqüência aumentou no início da fase lútea e manteve-se baixa na metade do período. Resultados semelhantes foram obtidos por Tsukamura (1998), o qual apontou que a freqüência dos pulsos e sua amplitude são variáveis que dependem da fase do ciclo estral.
Assim, nos ruminantes, existem evidências que mostram que a $\mathrm{P}_{4}$ inibe a freqüência de pulsos de LH e que o estradiol age principalmente para inibir a amplitude de pulso de LH, apesar de poder ocorrer uma certa sinergia entre esses dois hormônios (Price, 1999). Em diversas estruturas do SNC, como o córtex, sistema límbico, hipocampo e cerebelo, têm sido identificados receptores estrógeno-específicos. Acredita-se que os estrógenos atuem pela interação com os receptores do núcleo e de membrana celulares. Sua ligação com receptores nucleares possibilitaria uma ação ativadora/inibitória genética bastante diversificada (Soares et al., 2002). Em ovelhas, Arreguin-Arévalo e Nett (2006) verificaram que a inibição do LH pelo $E_{2}$ ocorre pela via dos receptores localizados na membrana celular.

A concentração de $\mathrm{P}_{4}$ plasmática é apresentada na Fig. 1. A concentração de $\mathrm{P}_{4}$ não diferiu $(\mathrm{P}>0,05)$ entre os grupos controle e tratado, com valores médios de $0,12 \pm 0,01$ e $0,14 \pm 0,01 \mathrm{ng} / \mathrm{ml}$; e $0,21 \pm 0,01$ e $0,19 \pm 0,01 \mathrm{ng} / \mathrm{ml}$ nos dias -2 e -1 antes da ovulação, respectivamente, de forma semelhante aos resultados de Campbell et al. (1990).

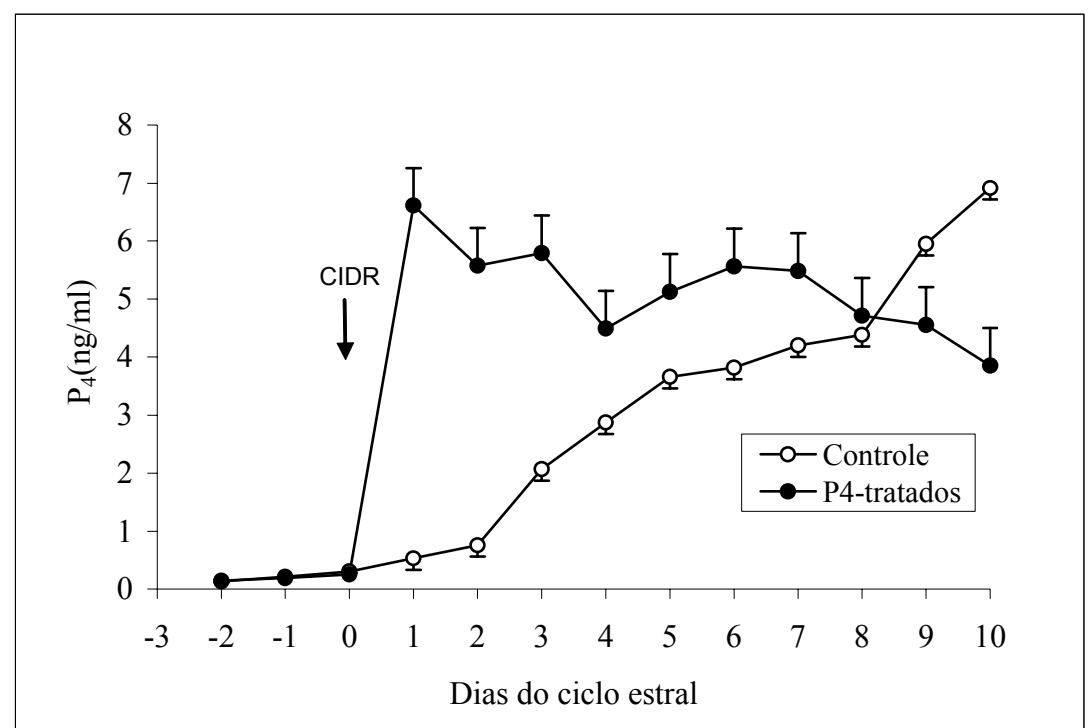

Figura 1. Concentrações plasmáticas de progesterona (médias \pm erro-padrão da média) em fêmeas ovinas Bergamácia tratadas ou não com progesterona exógena (CIDR) no dia da ovulação (dia zero). 
As ovelhas do tratamento-controle apresentaram $\mathrm{P}_{4}$ baixa no primeiro dia após a ovulação, $0,53 \pm 0,17 \mathrm{ng} / \mathrm{ml}$, e aumento $(\mathrm{P}<0,001)$ no terceiro dia, $2,07 \pm 0,17 \mathrm{ng} / \mathrm{ml}$. O aumento foi progressivo desde o quinto, $3,66 \pm 0,17 \mathrm{ng} / \mathrm{ml}$, até o $10^{\circ} \mathrm{dia}, 6,91 \pm 0,17 \mathrm{ng} / \mathrm{ml}$, o que evidenciou uma diferença $(\mathrm{P}<0,001)$ entre os dias estudados. Dessa forma, os resultados obtidos no grupocontrole confirmam as observações realizadas por González-Reyna et al. (1991).

Nas fêmeas controle, a concentração de $\mathrm{P}_{4}$ apresentou valores médios de $0,30 \pm 0,02 \mathrm{ng} / \mathrm{ml}$ no dia de emergência da primeira onda folicular ($0,71 \pm 0,7)$ a $3,82 \pm 0,17 \mathrm{ng} / \mathrm{ml}$ no dia do diâmetro folicular máximo $(5,5 \pm 0,5)$. Nas tratadas com a $\mathrm{P}_{4}$ exógena, a concentração de $\mathrm{P}_{4}$ foi de $0,25 \pm 0,02 \mathrm{ng} / \mathrm{ml}$ no dia de emergência folicular $(0,0 \pm 0,6)$, com aumentos até $5,56 \pm 0,56 \mathrm{ng} / \mathrm{ml}$ no dia do máximo crescimento $(4,0 \pm 0,4)$. Assim, observa-se que existe, dentro de cada onda de crescimento folicular, uma relação inversa entre a concentração plasmática de $\mathrm{P}_{4} \mathrm{e}$ o diâmetro do folículo dominante. $\mathrm{A}_{4}$ exógena liberada desde o CIDR exerceria um efeito de feedback negativo, inibindo a liberação de GnRH desde o hipotálamo e hipófise, regulando a liberação das gonadotrofinas (Martin, 1984), o que desencadearia aumentos significativos na concentração de $\mathrm{P}_{4}$.

\section{CONCLUSÕES}

Os efeitos inibitórios de $\mathrm{P}_{4}$ exógena na dinâmica folicular ovina foram mediados pelas alterações observadas na freqüência dos pulsos de LH. O mecanismo dessa inibição pode ter efeito nos programas de sincronização do estro e da ovulação, assim como os protocolos envolvendo superovulação em pequenos ruminantes.

\section{REFERÊNCIAS BIBLIOGRÁFICAS}

ALECOZAY, A.; SELCER, K.W.; CLARK, J.R. et al. Pattern of ovarian progesterone secretion during the luteal phase of the ovine estrous cycle. Biol. Reprod., v.39, p.287-94, 1988.

ARREGUIN-AREVALO, J.; NETT, T. A nongenomic actino of estradiol as the mechanism underlying the acute supresión of secretion of LH in ovariectomized ewes. Biol. Reprod., v.74, p.202-08, 2006.
CAMPBELL, B.K.; MANN, G.E.; McNEILLY, A.S. et al. Pulsatile secretion of inhibin, oestradiol and androstenedione by the ovary of the sheep during the oestrus cycle. $J$. Endocrinol., v.126, p.385-393, 1990.

CASTONGUAY, F.; DUFOUR, J.J.; MINVIELLE, F. et al. Follicular dynamics and dominance in Boorola $\mathrm{x}$ Finnish Landrace and Boorola x Suffolk ewes heterozygous for the F gene. J. Reprod. Fertil., v.89, p.193-203, 1990.

CURRIE, W.D.; MEDHAMURTHY, R.J.; COOK, S.J. et al. Seasonal fluctuation in diurnal rhythms of luteinizing hormone secretion in ewes during the mid-luteal phase of the oestrus cycle. J. Reprod. Fertil., v.97, p.71-74, 1993.

DOWNING, J.A.; JOSS, J.; SCARAMUZZI, R.J. Ovulation rate and the concentrations of $\mathrm{LH}$, $\mathrm{FSH}, \mathrm{GH}$, prolactin and insulin in ewes infused with tryptophan, tyrosine or tyrosine plus phenyalanine during the luteal phase of the oestrus cycle. Anim. Reprod. Sci., v.45, p.283297, 1997.

DRIANCOURT, M.A.; CAHILL, L.P. Preovulatory follicular events in sheep. $J$. Reprod. Fertil., v.71, p.205-211, 1984.

DRIANCOURT, M.A.; GIBSON, W.R.; CAHILL, L.P.Follicular dynamics throught the oestrus cycle in sheep. A review. Reprod. Nutr. Dev., v.25, p.1-15, 1985.

FORSBERG, M.; TAGLE, R.; MADEJ, A.; et al. Radioimmunoassay of bovine, ovine and porcine luteinising hormone with a monoclonal antibody and a human tracer. Act. Vet. Scand., v.34, p.255262, 1993.

FORTUNE, J.E. Ovarian follicular growth and development in mammals. Biol. Reprod., v.50, p.225-232, 1994.

GINTHER, O.J. Influence of progesterone and number of corpora lutea on ovaries in sheep. Am. J. Vet. Res., v.32, p.1987-1992, 1971.

GONZÁLEZ-REYNA, A.; VALENCIA, M.J.; FOOTE, W.C. et al. Hair sheep in México: reproduction in the Pelibuey sheep. Anim. Breed. Abstr., v.59, p.509-524, 1991.

GOODMAN, R.L.; KARSCH, F.J. Pulsatile secretion of luteinizing hormone: Differential suppression by ovarian steroids. Endocrinology, v.107, p.1286-1290, 1980. 
GOODMAN, R.L.; BITTMAN, E.L.; FOSTER, D.L. et al. Alterations in the control of luteinizing hormone pulse frequency underline the seasonal variation in estradiol negative feedback in the ewe. Biol. Reprod., v.27, p.580$590,1982$.

HARESIGN, W.; COOPER, A.C.; KHALID, M. et al. Patterns of gonadotropin secretion in cyclic Finn ewes selected for low and again high ovulation rate. Anim. Sci., v.61, p.251-257, 1995.

KARSCH, F.J.; LEGAN, S.J.; HAUGER, R.L. et al. Negative feedback action of progesterone on tonic luteinizing hormone secretion in the ewe: Dependence on the ovaries. Endocrinology, v.101, p.800-806, 1977.

KLEIN, P.; HERZOG, A.G. Hormonal effects on epilepsy in woman. Epilepsia, v.39, p.9-16, 1998.

KRUIP, A.M.; BRAND, A. Follicular growth during the normal cycle and after treatment with progestagens in the ewe. Ann. Biol. Anim. Biochem. Biophys., v.15, p.191-204, 1975.

LATOUR, D.; LITTELL, R. Advanced general lineal models with an emphasis on mixed models. Version 6.12. Cary: SAS Institute, 1996. 268p.

LEYVA, V.; BUCKRELL, B.C.; WALTON, J.S. Regulation of follicular and ovulation in ewes by exogenous progestagen. Theriogenology, v.50, p.395-416, 1998.

MARTIN, G.B. Factors affecting the secretion of luteinizing hormone in the ewe. Biol. Rev., v.59, p.1-87, 1984.

McNATTY, K.P.; HUDSON, N.L.; HENDERSON, K.M.; et al. Changes in gonadotrophin secretion and ovarian antral follicular activity in seasonally breeding sheep throught the year. J. Reprod. Fertil., v.70, p.309$321,1984$.

NOEL, B.; BISTER, J.L.; PIERQUIN, B. et al. Effects of FGA and PMSG on follicular growth and LH secretion in Suffolk ewes. Theriogenology, v.41, p.719-727, 1994.

NORRIS, T.A.; WEESNER, G.D.; SHELTON, J.M. et al. Biological activity of LH during the peripartum period and the estrous cycle of the ewe. Domest. Anim. Endocrinol., v.61, p.25-33, 1989.

PRICE, C.A. Secreção pulsátil de LH alterada durante a superovulação em bovinos. Arq. Bras. Fac. Vet. UFRGS, v.27, p.35-53, 1999.

RAVINDRA, J.P.; RAWLINGS, N.C.; EVANS, A.C.O. et al. Ultrasonographic study of ovarian follicular dynamics in ewes during the estrous cycle. J. Reprod. Fertil., v.101, p.501-509, 1994.

RUBIANES, E.; CASTRO, T.; CARBAJAL, B. Effect of high progesterone levels during the growing phase of the dominant follicle of wave 1 in ultrasonically monitored ewes. Can. J. Anim. Sci., v.76, p.1-3, 1996.

SOARES, C.N.; PROUTY, J.; POITRAS, J. Ocorrência e tratamento de quadros depresivos por hormônios sexuais. Rev. Bras. Psiquiatr., v.24, p.48-54, 2002.

THIÉRY, J.C.; MARTIN, G.B. Neurophysiological control of the secretion of gonadotrophin-releasing hormone and luteinizing hormone in sheep -A review. Reprod. Fertil. Dev., v.3, p.137-173, 1991.

TSUKAMURA, H. Neuroendocrine mechanism regulating luteinizing hormone secretion during periovulatory period: Negative and positive feedback actions of estrogen in the brain. $J$. Reprod. Dev., v.44, p.j81-j90, 1998. 